\section{International Scientific Journal Theoretical \& Applied Science}

Nozim Muhammadrashitovich Kurbonov

Senior Researcher,

Centre for the development of software and hardware-program complex, Tashkent, Uzbekistan nozim_kurbonov@mail.ru

Year: 2017 Issue: 05 Volume: 49

Published: $30.05 .2017 \quad$ http://T-Science.org

SECTION 2. Applied mathematics. Mathematical modeling.

\title{
COMPUTATIONAL EXPERIMENT FOR STUDYING COFILTERING OF LIQUID AND GAS IN A POROUS MEDIUM
}

\begin{abstract}
The paper deals with a mathematical model and results of numerical experiments for studying the process of "oil-gas-water" mixture filtration in a porous medium to make concrete practical recommendations and administrative decisions. The developed mathematical support allows to define duration of reservoir operation time, depending on its length and capacity, number of wells and their production rates. Numerical calculations defined important parameters of the process their ranges. Results of numerical experiments were presented as graphics. On the basis of material balance equation there was shown suitability of the developed mathematical and software for calculation of pressure fields and saturation, as well as for calculating field development indicators in the "oil-gas", "oil-water" and "oil-gas-water" systems.

Key words: mathematical model, numerical method, numerical simulation, filtration, liquid, oil, gas, porous media, fluid software tool.

Language: Russian

Citation: Kurbonov NM (2017) COMPUTATIONAL EXPERIMENT FOR STUDYING COFILTERING OF LIQUID AND GAS IN A POROUS MEDIUM. ISJ Theoretical \& Applied Science, 05 (49): 1-7.

Soi: http://s-o-i.org/1.1/TAS-05-49-1 Doi: crossef https://dx.doi.org/10.15863/TAS.2017.05.49.1

\section{ВЫЧИСЛИТЕЛЬНЫЙ ЭКСПЕРИМЕНТ ДЛЯ ИССЛЕДОВАНИЯ ПРОЦЕССА СОВМЕСТНОЙ ФИЛЬТРАЦИИ ЖИДКОСТЕЙ И ГАЗА В ПОРИСТОЙ СРЕДЕ}

Аннотация: В работе приведены математическая модель и результаты вычислительных экспериментов на ЭВМ для исследования процесса фильтрации смеси «нефть-газ-вода» в пористой среде с целью принятия конкретных практических рекомендачий и управленческих решений. Разработанный математический инструментарий позволяет определять время эксплуатации пласта в зависимости от его длины и мощуности, числа скважин и их дебитов. Численными расчетами были установлены важнейшие параметры исследуемого процесса и диапазоны их изменения. Результаты проведенных вычислительных экспериментов на ЭВМ представлены в виде графических объектов. На основе уравнения материального баланса показана пригодность разработанного математического и программного обеспечения для расчетов полей давлений и насыщенностей, а также показателей разработки месторождений в системах «нефть-газ», «нефть-вода», «нефть-газ-вода».

Ключевые слова: математическая модель, численный метод, вычислительный эксперимент, фильтрация, жидкость, нефть, газ, пористая среда, флюид, программное средство.

\section{Введение}

В современном мире такие энергетические ресурсы, как нефть и газ играют ведущую роль в развитии экономики любой страны. В значительной степени, масштаб потребления энергоресурсов определяет уровень развития производительных сил каждого государства.

Качественное развитие нефтегазового комплекса традиционно связано с применением методов компьютерного моделирования и комплексного исследования объекта с целью определения основных показателей нефтегазовых месторождений и принятия управленческих решений. В этой связи, остается актуальной проблема разработки новых и усовершенствования существующих математических моделей и вычислительных алгоритмов для исследования сложных процессов, происходящих при разнообразных 
естественных и искусственных условиях воздействия на залежи полезных ископаемых.

В данном направлении работают многочисленные исследователи, как в Узбекистане, так и за рубежом, которыми уже получены значительные результаты практического и теоретического характера.

В частности, Ф. Бойер [1] описывает некоторые аспекты моделирования диффузного потока несжимаемых сред, состоящих из трех несмешивающихся компонентов, без фазовых превращений. Моделирование трехфазных потоков дополнено путем включения системы Cahn-Hilliard и уравнений Навье-Стокса, где поверхностное натяжение принимается во внимание через объемные капиллярные силы. Уравнения дискретизируются по времени и пространству, обращая внимание на то, что большая часть основных свойств исходной модели, таких как сохранение объема и оценка энергии, должны быть сохранены на дискретном уровне. Адаптивный метод уточнения используется для получения решения для очень тонких движущихся внутренних слоев, ограничивая при этом общее количество узлов в сетке дискретной модели.

В работе [2] представляется стабильная численная схема для моделирования многофазного потока в пористых средах, где характерный размер области течения составляет от микрон до миллиметров. Численный метод разработан для эффективного моделирования многофазных потоков в пористых средах со сложным характером движения границы раздела фаз и нерегулярных твердых границ. Уравнения Навье-Стокса дискретизированы с использованием подхода конечного объема. Капиллярные силы вычисляются с использованием модели полупроницаемой поверхностной силы, в которой область перехода для капиллярного давления эффективно ограничена одним узлом сетки. Эта новая формулировка вместе с двумя новыми методами фильтрации, разработанных для коррекции капиллярных сил, позволяет проводить моделирование при очень низких капиллярных числах и избегать наличия нефизических скоростей. Капиллярные силы реализуются с использованием полунеявных композиций, что позволяет задавать большие размеры временных шагов при малых капиллярных числах. Точность и стабильность численного метода проверены на нескольких тестовых задачах, результаты решения которых указывают на потенциал метода для прогнозирования процессов многофазного потока.

А.В. Цепаевым [3] решена задача многофазной фильтрации жидкостей при наличии скважин. Автором представлены алгоритмы, основанные на методах декомпозиции задач для MPI платформы. Предложенные алгоритмы реализованы на высокопроизводительной вычислительной системе, комбинирующей мощности CPU и GPU.

Т.С. Иманкулов и Д.Ж. Ахмед-Заки [4] рассматривают двухмерную математическую модель многофазной многокомпонентной фильтрации жидкости в пласте с учетом капиллярных и гравитационных сил, термодинамических свойств фаз и компонентов. Расчет фазового равновесия производится авторами с использованием метода констант фазового равновесия. Предложенная модель и алгоритм ее решения реализованы в виде программного средства для проведения вычислительных экспериментов на ЭВМ. В ходе расчетов были получены поля распределений давления и насыщенности фаз, изменения плотностей, концентрации компонент в водной, нефтяной и газовой фазе.

Численный метод решения обратной задачи определения дебитов скважин по заданным забойным давлениям для многомерной модели течения слабосжимаемой жидкости в упругодеформируемой пористой среде предложен авторами работы [5]. Аппроксимацию по пространственным переменным авторы выполняли методом конечных элементов, что позволило использовать неструктурированные сетки со сгущением в окрестностях расположения скважин. А дискретизация по времени построена с использованием неявной разностной аппроксимации. Авторами приведены результаты численных решений для двумерной и трехмерной постановок указанной задачи.

Н.Р. Батраков и др. в статье [6] приводят математическую модель трехфазной трехкомпонентной фильтрации системы «сверхкритический флюид-вода-нефть» в диапазоне проницаемостей пласта менее 0,01 Дарси, при обводненности пласта более 90 \%, вязкости нефти более 60 мПа. Предложенная авторами модель описывает процесс вытеснения нефти из обводненного пласта сверхкритическим диоксидом углерода с учетом наличия пороговых ограничений, свойственных традиционным методам увеличения нефтеотдачи.

М.А. Трапезникова, Н.Г. Чурбанова и А.А. Люпа посвятили работу [7] развитию математической модели течения слабосжимаемой жидкости в пористой среде, построенной по аналогии с квазигазодинамической системой уравнений. Модель обобщена для случая трехфазной жидкости и дополнена уравнением сохранения энергии, что позволяет использовать ее при моделировании перспективных термических методов нефтедобычи. 


\begin{tabular}{|c|c|c|c|c|c|c|}
\hline Impact Factor: & $\begin{array}{l}\text { ISRA (India) } \\
\text { ISI (Dubai, UAE } \\
\text { GIF (Australia) } \\
\text { JIF }\end{array}$ & $\begin{array}{l}=1.344 \\
=0.829 \\
=0.564 \\
=1.500\end{array}$ & $\begin{array}{l}\text { SIS (USA) } \\
\text { PИНЦ (Russia) } \\
\text { ESJI (KZ) } \\
\text { SJIF (Morocco) }\end{array}$ & $\begin{array}{l}=0.912 \\
=0.234 \\
=3.860 \\
=\mathbf{2 . 0 3 1}\end{array}$ & $\begin{array}{l}\text { ICV (Poland) } \\
\text { PIF (India) } \\
\text { IBI (India) }\end{array}$ & $\begin{array}{l}=6.630 \\
=1.940 \\
=4.260\end{array}$ \\
\hline
\end{tabular}

Д.Я. Исуповым и др. [8] численно исследованы процессы течения многофазной жидкости в слоистой пористой среде с учетом капиллярных и гравитационных сил. Предложенные авторами вычислительные алгоритмы были апробированы на тестовых задачах о двух- и трехфазных течениях. Описанный в указанной работе подход может быть использован для решения экологических задач, связанных с загрязнением почвы и грунтовых вод вследствие разлива нефтепродуктов и других загрязнителей.

К.Ю. Богачевым в исследовании [9] предложена математическая модель трещиноватости, возникающей вокруг скважин при проведении гидравлического разрыва пласта или при превышении давления закачки. Вычислительный алгоритм решения задачи разработан для гибридной MPI платформы. Результаты экспериментов на кластере демонстрируют ускорение решения более чем в 50 раз, по сравнению с последовательным алгоритмом. Полученные автором результаты могут быть использованы при численном решении задач разработки углеводородного сырья, а также при решении других систем уравнений в частных производных на параллельных ЭВМ.
Моделирование нестационарного течения многофазного потока - «нефть-газ-вода» в пласте и скважине при работе насоса рассматривается в [10]. Авторы принимают течение в пласте однофазным, и учитывают относительное движение компонентов многофазной среды, тепло- и массообмен, а также режим течения. Приведены примеры использования модели для иллюстрации нестационарных эффектов, возникающих в процессе добычи нефти.

Анализ вышеуказанных источников и других научных работ показал, что в исследованиях практически не рассмотрен процесс двухстороннего вытеснения нефти газом и водой, в результате которого образуются зоны чистого газа, смеси «нефть-газ-вода» и чистой нефти. В настоящей работе предприняты усилия для восполнения данного пробела.

\section{Постановка задачи}

Для разработки и проектирования нефтегазовых месторождений и определения основных параметров их значений, необходимо рассмотреть совместную фильтрацию нефти, воды и газа в пористой среде, которая описывается следующей системой нелинейных дифференциальных уравнений в безразмерном виде:

$$
\left\{\begin{array}{l}
\frac{\partial}{\partial x}\left(\mathrm{~K}_{\Gamma} \frac{\partial P}{\partial x}\right)+\frac{\partial}{\partial y}\left(K_{\Gamma} \frac{\partial P}{\partial y}\right)=\frac{\partial}{\partial t}\left(P S_{\Gamma}\right)+B \frac{\partial}{\partial t}\left(S_{\mathrm{H}}+B_{\mathrm{BH}} S_{\mathrm{B}}\right) \\
\frac{\partial}{\partial x}\left(K_{\mathrm{H}} \frac{\partial P}{\partial x}\right)+\frac{\partial}{\partial y}\left(K_{\mathrm{H}} \frac{\partial P}{\partial y}\right)=B \frac{\partial S_{\mathrm{H}}}{\partial t} \\
\frac{\partial}{\partial x}\left(K_{\mathrm{B}} \frac{\partial P}{\partial x}\right)+\frac{\partial}{\partial y}\left(K_{\mathrm{B}} \frac{\partial P}{\partial y}\right)=\frac{\mu_{\mathrm{B}}}{\mu_{\Gamma}} B \frac{\partial S_{\mathrm{B}}}{\partial t} \\
S_{\mathrm{H}}+S_{\mathrm{B}}+S_{\Gamma}=1
\end{array}\right.
$$

со следующими начальными

$$
\begin{gathered}
P(x, y, 0)=P^{0}(x, y), S_{\mathrm{H}}(x, y, 0)=S_{\mathrm{H}}^{0}(x, y), \\
S_{\mathrm{B}}(x, y, 0)=S_{\mathrm{B}}^{0}(x, y), S_{\Gamma}(x, y, 0)=S_{\Gamma}^{0}(x, y),(x, y) \in G,
\end{gathered}
$$

граничными и внутренними условиями

$$
\begin{aligned}
& \alpha \frac{\partial P}{\partial n}+\beta\left(P-P_{K}\right)=0, \quad x, y \in \Gamma, \\
& 2 \pi b_{i} R_{c j} \int K \frac{\partial P}{\partial n} d \sigma=q_{j}, j=\overline{1, n},
\end{aligned}
$$

где

$$
K=K_{\mathrm{H}}+A_{\Gamma \mathrm{H}} K_{\Gamma} P+A_{\mathrm{BH}} K_{\mathrm{B}}, A_{\Gamma \mathrm{H}}=\frac{P_{\mathrm{H}} \mu_{\mathrm{H}}}{\rho_{\mathrm{H}} R Z T \mu_{\Gamma}}, A_{\mathrm{BH}}=\frac{\rho_{\mathrm{B}} \mu_{\mathrm{H}}}{\rho_{\mathrm{H}} \mu_{\mathrm{B}}}, B=\frac{\rho_{\mathrm{H}} R Z T}{P_{\mathrm{H}}}, \quad B_{\mathrm{BH}}=\frac{\rho_{\mathrm{B}}}{\rho_{\mathrm{H}}} .
$$

Искомая функция ищется в области изменения

$$
\Omega=\{0 \leq x \leq 1\} \cup\{0 \leq y \leq 1\} \cup\{0 \leq t \leq T\}, t=\frac{\rho_{\mathrm{H}} K R Z T}{m \mu_{\mathrm{H}} L^{2}} \tau .
$$

Здесь $\quad K_{\mathrm{H}}, K_{\Gamma}, K_{\mathrm{B}} \quad$ и $\quad \mu_{\mathrm{H},} \mu_{\Gamma,} \mu_{\mathrm{B}}$, соответственно фазовые проницаемости и вязкости нефти, газа и воды; $P$ - давление;
$S_{\mathrm{H}}, S_{\Gamma}, S_{\mathrm{B}}$ - насыщенности породы нефтью и газом и водой; $m$ - пористость; $K$ - абсолютная проницаемости породы; $\sigma$ - контур скважины; 


\begin{tabular}{|c|c|c|c|c|c|c|}
\hline Impact Factor: & $\begin{array}{l}\text { ISRA (India) } \\
\text { ISI (Dubai, UAB } \\
\text { GIF (Australia) } \\
\text { JIF }\end{array}$ & $\begin{array}{r}=1.344 \\
=0.829 \\
=0.564 \\
=1.500\end{array}$ & $\begin{array}{l}\text { SIS (USA) } \\
\text { PИHЦ (Russia) } \\
\text { ESJI (KZ) } \\
\text { SJIF (Morocco) }\end{array}$ & $\begin{array}{l}=0.912 \\
=0.234 \\
=3.860 \\
=\mathbf{2 . 0 3 1}\end{array}$ & $\begin{array}{l}\text { ICV (Poland) } \\
\text { PIF (India) } \\
\text { IBI (India) }\end{array}$ & $\begin{array}{l}=6.630 \\
=1.940 \\
=4.260\end{array}$ \\
\hline
\end{tabular}

Г - граница пласта; $P_{K}$ - давление на границе пласта; $\quad P^{0}(x, y), \quad S_{\mathrm{H}}^{0}(x, y), \quad S_{\Gamma}^{0}(x, y), S_{\mathrm{B}}^{0}(x, y) \quad-$ соответственно начальное распределение давления, нефтенасыщенностей и газонасыщенностей; $n$ - число скважин; $\rho_{\Gamma}, \rho_{\mathrm{H}}, \rho_{\mathrm{B}}-$ соответственно плотность газа, нефти и воды; $R$ - газовая постоянная; $T$ - абсолютная температура; $Z$ - сжимаемость газа; $P_{\text {н }}$ начальное пластовое давление.

Относительные фазовые проницаемости являются функцией двух насыщенностей $K_{\mathrm{H}}\left(S_{\mathrm{H}}, S_{\Gamma}\right), K_{\Gamma}\left(S_{\mathrm{H}}, S_{\Gamma}\right), K_{\text {в }}\left(S_{\mathrm{H}}, S_{\Gamma}\right)$ определяются на основе истории разработки или обработкой результатов эксперимента.

Для аппроксимации краевой задачи введем пространственную и временную сетки следующим образом:

$$
\begin{gathered}
\omega_{h_{i}, h_{j}}=\left\{x_{i}=x_{i-1}+h_{i}, i=\overline{1, N_{1}}, y_{i}=y_{i-1}+h_{j}, j=\overline{1, N_{2}}\right\}, \\
\omega_{\tau k}=\left\{t_{k}=t_{k-1}+\tau_{k}, k=1,2, \ldots\right\} .
\end{gathered}
$$

Здесь $h_{i}, h_{j} \quad$ - значения шагов пространственной сетки, соответствующие узлу с координатами $x_{i}, x_{j} ; \tau_{k}$ - значения шага сетки для момента времени $t_{k}$. Будем считать, что внешняя граница области фильтрации может быть аппроксимирована в ступенчатом виде. Тогда дискретная область фильтрации может быть рассмотрена как совокупность узлов семейства прямых линий $c_{1 i, j}=\overline{1, N_{2}}$ параллельных оси $\mathrm{OX}$ или как совокупность узлов семейства прямых линий $c_{2 i, j}=\overline{1, N_{1}}$ параллельных оси ОY.

Метод решения и обсуждение результатов. Для решения задачи применяем схему продольно-поперенчных направлений и получем системы уравнений в конечных разностях для внутренних узлов дискретной области фильтрации на прямых, которые решаются методом прогонки [11-14]. Так как задача нелинейная, то для решения используем итерационный метод, а сходимость проверяем с помощью

$$
\max _{j}\left|P_{i j}^{(S+1)}-P_{i j}^{(S)}\right|<\varepsilon,
$$

где $S$-номер итерации, $\varepsilon$-заданная точность.

На основе указанного алгоритма было разработано программное средство для анализа динамического состояния объекта с учетом совместного движения трехфазной среды при различных условиях функционирования пластовой системы.

Результаты проведенных вычислительных экспериментов на ЭВМ приведены на рис. 1-6. В расчетах принимали область фильтрации в форме квадрата, круга и эллипса при 5 функционирующих скважинах.

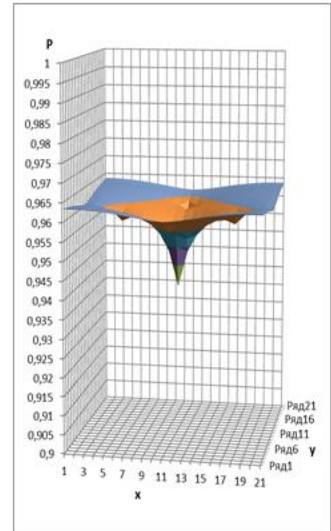

а) при $\tau=132$ сут.

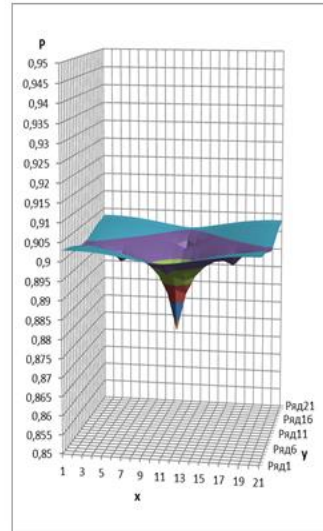

б) при $\tau=332$ сут.

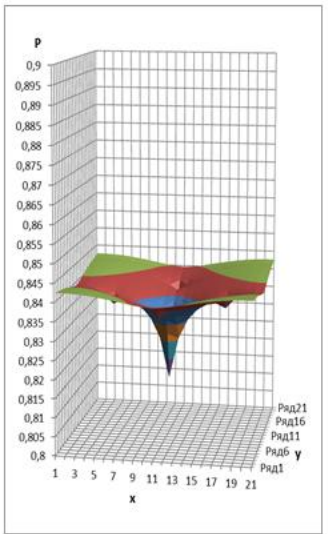

в) при $\tau=532$ сут.

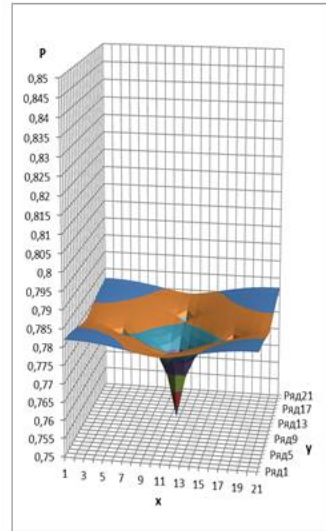

г) при $\tau=732$ сут.

Рисунок 1 - Трехмерная визуализация перераспределения давления газа в пласте по времени (когда область квадрат). 


\begin{tabular}{l|lrl|l|ll} 
& ISRA (India) & $=\mathbf{1 . 3 4 4}$ & SIS (USA) & $=\mathbf{0 . 9 1 2}$ & ICV (Poland) & $=\mathbf{6 . 6 3 0}$ \\
Impact Factor: & ISI (Dubai, UAE) $=\mathbf{0 . 8 2 9}$ & PUHU (Russia) $=\mathbf{0 . 2 3 4}$ & PIF (India) & $=\mathbf{1 . 9 4 0}$ \\
& GIF (Australia) & $\mathbf{0 . 5 6 4}$ & ESJI (KZ) & $=\mathbf{3 . 8 6 0}$ & IBI (India) & $\mathbf{4 . 2 6 0}$
\end{tabular}

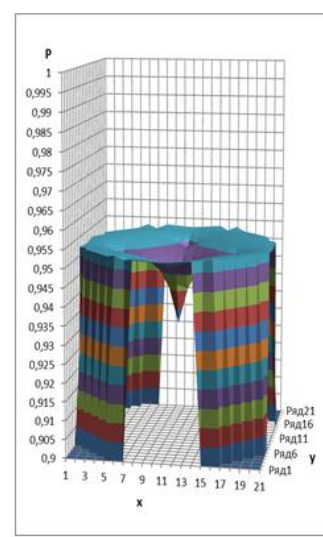

a) при $\tau=132$ сут.

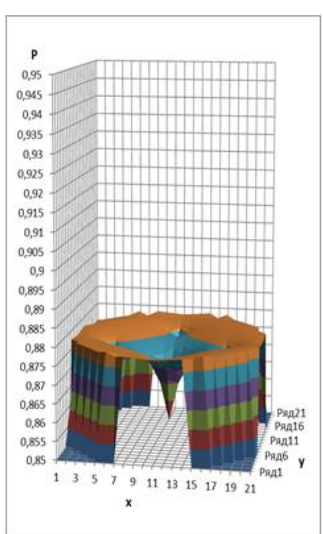

б) при $\tau=332$ сут.

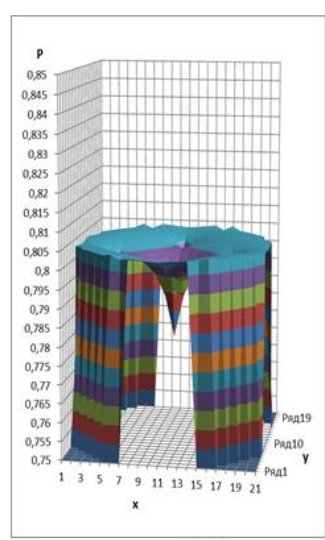

в) при $\tau=532$ сут.

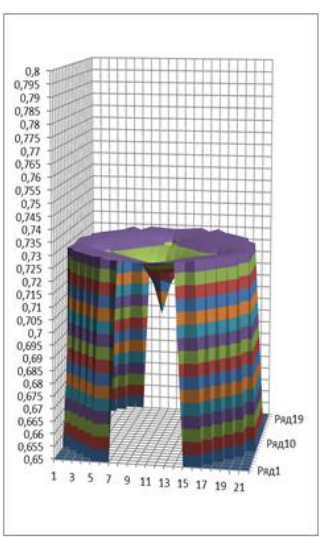

г) при $\tau=732$ сут.

Рисунок 2 - Трехмерная визуализация перераспределения давления газа в пласте по времени (когда область круг).

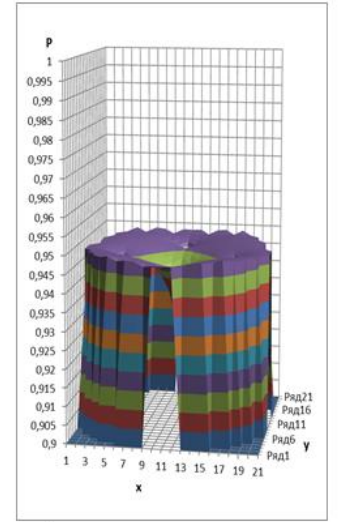

a) при $\tau=132$ сут.

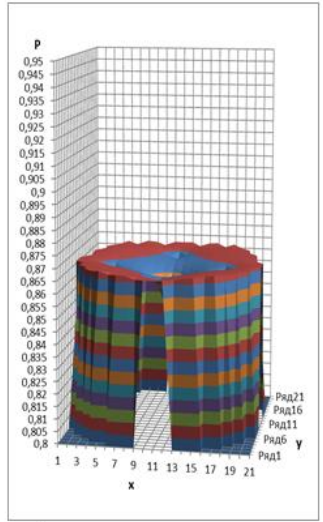

б) при $\tau=332$ сут.

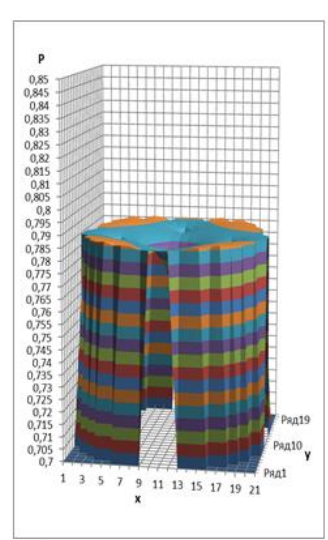

в) при $\tau=532$ сут.

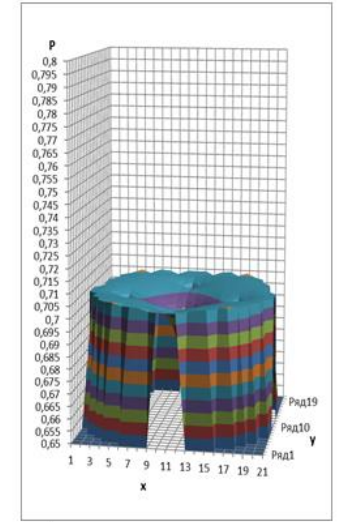

г) при $\tau=732$ сут.

Рисунок 3 - Трехмерная визуализация перераспределения давления газа в пласте по времени (когда область эллипс).

Как видно из рисунков $1-3$, значение давления газа вокруг скважины плавно уменьшается при постоянном коэффициенте

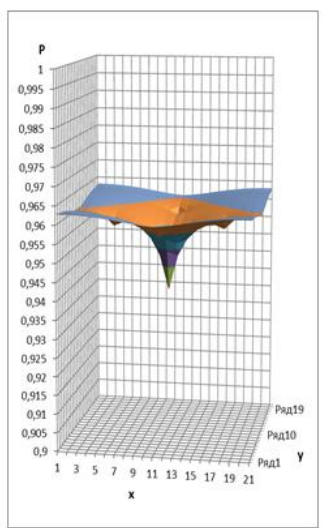

a) при $\tau=132$ сут.

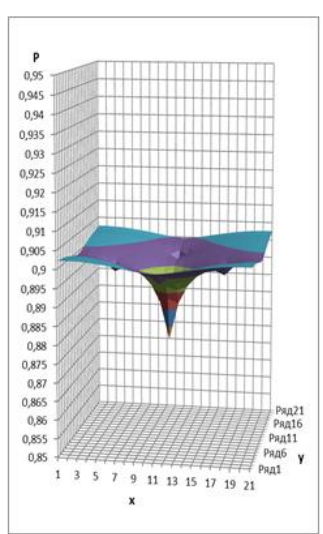

б) при $\tau=332$ сут.

фильтрации, а уменьшение давления распространяется в рассматриваемой области с течением времени.

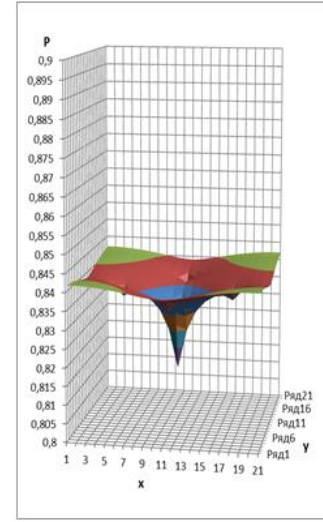

в) при $\tau=532$ сут.

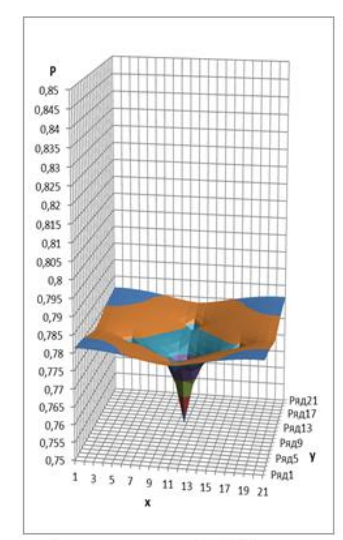

г) при $\tau=732$ сут.

Рисунок 4 - Трехмерная визуализация перераспределения давления нефти в пласте по времени (когда область квадрат). 


\begin{tabular}{l|lrl|l|ll} 
& ISRA (India) & $=\mathbf{1 . 3 4 4}$ & SIS (USA) & $=\mathbf{0 . 9 1 2}$ & ICV (Poland) & $=\mathbf{6 . 6 3 0}$ \\
Impact Factor: & ISI (Dubai, UAE) $=\mathbf{0 . 8 2 9}$ & PUHL (Russia) $=\mathbf{0 . 2 3 4}$ & PIF (India) & $=\mathbf{1 . 9 4 0}$ \\
& GIF (Australia) & $\mathbf{0 . 5 6 4}$ & ESJI (KZ) & $=3.860$ & IBI (India) & $=\mathbf{4 . 2 6 0}$ \\
& JIF & $\mathbf{1 . 5 0 0}$ & SJIF (Morocco) & $=\mathbf{2 . 0 3 1}$ & & \\
\hline
\end{tabular}

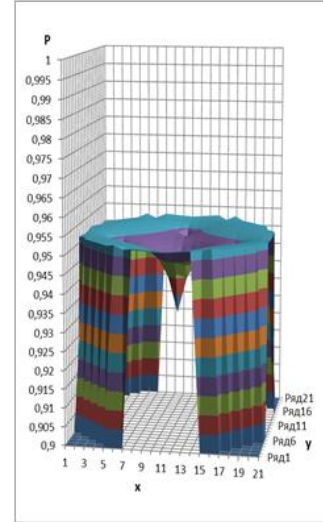

a) при $\tau=132$ сут.

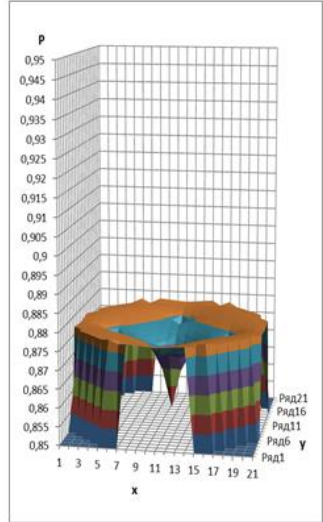

б) при $\tau=332$ сут.

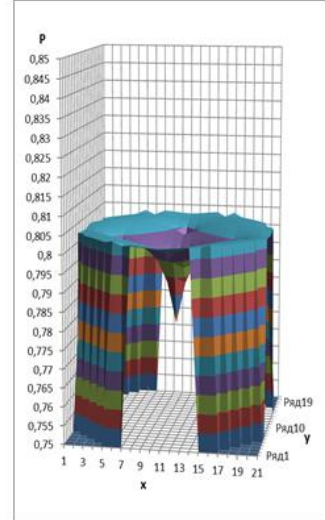

в) при $\tau=532$ сут.

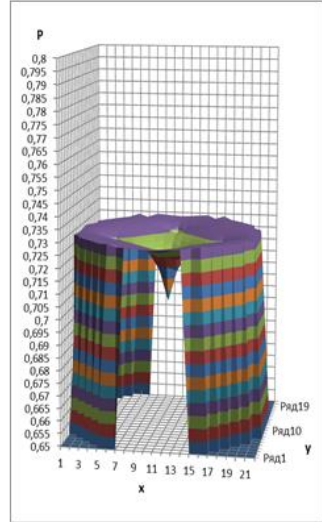

г) при $\tau=732$ сут.

Рисунок 5 - Трехмерная визуализация перераспределения давления нефти в пласте по времени (когда область круг).

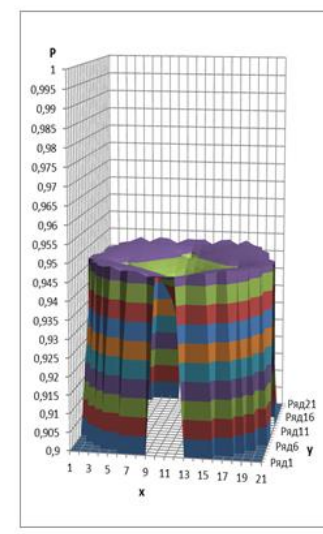

a) при $\tau=132$ сут.

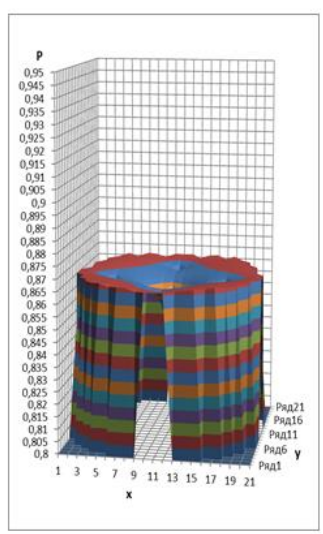

б) при $\tau=332$ сут.

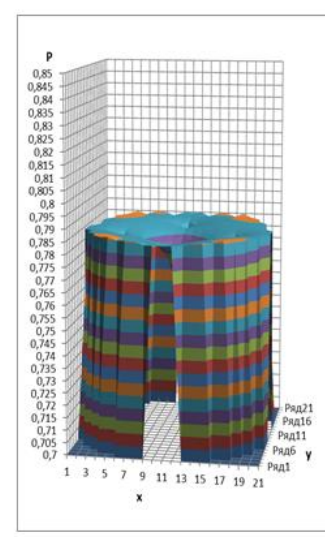

в) при $\tau=532$ сут.

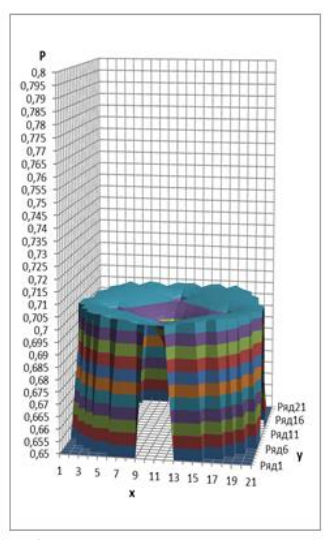

г) при $\tau=732$ сут.

Рисунок 6 - Трехмерная визуализация перераспределения давления нефти в пласте по времени (когда область эллипс).

Из рисунков 4 - 6 следует, что давление нефти вокруг скважины также плавно уменьшается при постоянном коэффициенте фильтрации, и уменьшение давления распространяется в рассматриваемой области с течением времени.

\section{Заключение}

Вычислительными экспериментами определены основные параметры процесса фильтрации смеси «нефть-газ-вода» в пористой среде и диапазоны их изменения, что может быть использовано с целью проектирования и разработки углеводородных месторождений при неустановившейся фильтрации флюидов в пористых средах.

Проведенными численными расчетами на ЭВМ установлено время эксплуатации пласта в зависимости от длины и мощности пласта, числа скважин и их дебитов.
Анализ результатов вычислительных экспериментов при широких изменениях фильтрационных параметров для решения различных тестовых задач, показывает адекватность построенных математических моделей, сходимость и устойчивость построенных вычислительных алгоритмов.

Результаты подтверждают пригодность алгоритма и программы для расчетов полей давлений и насыщенностей, а также показателей разработки месторождений в системах «нефтьгаз-вода».

Разработанные математическая модель, вычислительный алгоритм и программное средство могут быть использованы для анализа функционирования, оперативного управления и прогнозирования разработки нефтегазовых месторождений при различных условиях воздействия на пласт и принятия конкретных практических рекомендаций. 


\begin{tabular}{|c|c|c|c|c|c|c|}
\hline Impact Factor: & $\begin{array}{l}\text { ISRA (India) } \\
\text { ISI (Dubai, UAE } \\
\text { GIF (Australia) } \\
\text { JIF }\end{array}$ & $\begin{array}{l}=1.344 \\
=0.829 \\
=0.564 \\
=1.500\end{array}$ & $\begin{array}{l}\text { SIS (USA) } \\
\text { PИНЦ (Russia) } \\
\text { ESJI (KZ) } \\
\text { SJIF (Morocco) }\end{array}$ & $\begin{array}{l}=0.912 \\
=0.234 \\
=3.860 \\
=2.031\end{array}$ & $\begin{array}{l}\text { ICV (Poland) } \\
\text { PIF (India) } \\
\text { IBI (India) }\end{array}$ & $\begin{array}{l}=6.630 \\
=1.940 \\
=4.260\end{array}$ \\
\hline
\end{tabular}

\section{References:}

1. Boyer F, Lapuerta C, Minjeaud S, Piar B, Quintard M (2010) Cahn-Hilliard/NavierStokes Model for the Simulation of ThreePhase Flows // Transport in Porous Media. 2010. - Vol. 82, № 3. - p. 463-483 doi:10.1007/s11242-009-9408-z.

2. Ali Q. Raeini, Martin J. Blunt, Branko Bijeljic (2012) Modelling two-phase flow in porous media at the pore scale using the volume-offluid method // Journal of Computational Physics.-USA, 2012.- Vol. 231, № 17. - p. 5653-5668 doi>10.1016/j.jcp.2012.04.011

3. Cepaev AV (2013) Reshenie zadach trehfaznoj fil'tracii zhidkosti $\mathrm{V}$ trehmernyh plastah $\mathrm{S}$ gidrodinamicheski nesovershennymi skvazhinami na geterogennyh vychislitel'nyh sistemah // Fiziko-matematicheskie nauki, Uchen. zap. Kazan. un-ta. Ser. Fiz.-matem. nauki, 155, № 3, Izd-vo Kazanskogo un-ta, Kazan', 2013, 71-79.

4. Imankulov TS, Ahmed-Zaki DZ (2016) Chislennoe modelirovanie mnogofaznoj mnogokomponentnoj fil'tracii zhidkosti V poristoj srede // Vestnik KazNITU.-2016.— № 4(116). - p. 29-51.

5. Vabishhevich PN, Vasil'ev VI, Vasil'eva MV, Nikiforov DJ (2015) Chislennoe reshenie odnoj obratnoj zadachi fil'tracii // Uchenye zapiski kazanskogo universiteta. Serija: Fizikomatematicheskie nauki.- Kazan', 2015. - T. 157, № 4. p. 79-89.

6. Batrakov NR, Absaljamov RS, Galimzjanov RR, Zakiev ID, Radaev AV, Sabirzjanov AN, Muhamadiev AA (2013) Issledovanie processa vytesnenija nefti iz obvodnennogo plasta sverhkriticheskim dioksidom ugleroda // Vestnik Kazanskogo tehnologicheskogo universiteta. - Kazan', 2013. - T. 16, № 10. p. 245-247.

7. Trapeznikova MA, Churbanova NG, Lupa AA (2015) Three-phase fluid flow simulation in porous media, taking into account the thermal effects // Mathematica Montisnigri. - 2015. Vol. 33. - p. 105-115.
8. Isupov DJ, Trapeznikova MA, Churbanova NG, Shil'nikov EV (2010) Modelirovanie prosachivanija mnogofaznyh zhidkostej V sloistoj poristoj srede // Matematicheskoe modelirovanie. - Moskva, 2010. - T. 22, № 6. p. 84-98.

9. Bogachev KJ (2011) Jeffektivnoe reshenie zadachi fil'tracii vjazkoj szhimaemoj mnogofaznoj mnogokomponentnoj smesi na parallel'nyh JeVM: Avtoreferat diss. na soisk. Stepeni d. f-m.n. - Moscow, 2011.-38 p.

10. Topol'nikov AS, Bolotnova RH, Buzina VA, Agisheva UO (2014) Matematicheskoe modelirovanie dinamicheskih processov $\mathrm{V}$ neftedobyvajushhej skvazhine // Voprosy sovremennoj nauki i praktiki. Universitet im. V.I. Vernadskogo. 2014. № 4 (54). p. 112-118.

11. Sadullaev R, Bobokaev SN, Kurbonov NM, Shevcov VM (2009) Modelirovanie processov sovmestnoj mnogofaznoj fil'tracii zhidkostej i gaza v poristoj srede v plane // Sovmestnyj vypusk Uzbekskogo zhurnala Problemy informatiki i jenergetiki i Sbornika nauchnyh trudov Voprosy vychislitel'noj i prikladnoj matematiki. - Tashkent, 23-26 sentjabrja 2009. - p. 44-49.

12. Ravshanov N, Kurbonov N, Mukhamadiev A (2016) An Approximate Analytical Solution of the Problem of Fluid Filtration in the Multilayer Porous Medium // International Journal of Computational Methods. - 2016. - Vol. 13, № $6 . \quad 1650042$ [10 pages $]$ DOI: http://dx.doi.org/10.1142/S0219876216500420

13. Kurbonov NM, Saliev EA (2016) Computer experiment to study of filtration oil, gas and water in a porous medium // Problems of computational and applied mathematics. Tashkent, 2016. - № 1(3). -p. 13-22.

14. Ravshanov N, Kurbonov NM (2015) Computer modeling of the filtration process of fluids in porous media // Bulletin of South Ural State University. Series: Computational Mathematics and Informatics. - Chelyabinsk, 2015. - Vol. 4. - № 2. - p. 89-106. 